Journal of Advanced Research in Fluid Mechanics and Thermal Sciences

Journal homepage: www.akademiabaru.com/arfmts.html ISSN: 2289-7879

\title{
Hall Current and Thermal Radiation Effects on Heat and Mass Transfer of Unsteady MHD Flow of a Viscoelastic Micropolar Fluid through a Porous Medium
}

\author{
Bommanna Lavanya ${ }^{1, *}$ \\ Department of Mathematics, MIT MAHE, Manipal-576104, KA, India
}

\begin{tabular}{ll} 
ARTICLE INFO & ABSTRACT \\
\hline Article history: & The present paper is concerned to analyze the effect of hall current on heat and \\
Received 27 August 2019 & thermal radiation and mass transfer of unsteady MHD flow of a viscoelastic micropolar \\
Received in revised form 20 October 2019 & $\begin{array}{l}\text { fluid through a porous medium with chemical reaction. The governing partial } \\
\text { Accepted 22 November 2019 }\end{array}$ \\
Available online 20 March 2020 & $\begin{array}{l}\text { differential equations are transformed to dimensionless equations using dimensionless } \\
\text { verturbation technique. The effects of various governing parameters on the velocity, } \\
\text { temperature, concentration, skin-friction coefficient, Nusselt number and Sherwood } \\
\text { number are shown in figures and tables and analyzed in detail. }\end{array}$
\end{tabular}

Keywords:

MHD, Hallcurrent, Micropolar fluid,

Radiation, Chemical reaction

Copyright $@ 2020$ PENERBIT AKADEMIA BARU - All rights reserved

\section{Introduction}

The Magneto-Hydrodynamics (MHD) boundary layers with heat and mass transfer over flat surfaces are found in many engineering and geophysical applications such as geothermal reservoirs, thermal insulation, enhanced oil recovery, packed-bed catalytic reactors, cooling of nuclear reactors. Because of this reason, many researchers tend to apply the MHD flow into their problems. For instance, Khaleque and Samad [1] examined the effects of radiation heat generation and viscous dissipation on MHD free convection flow along a stretching sheet. Amkadni and Azzouzi [2] studied the similarity solution of MHD boundary layer flow over a moving vertical cylinder. Meanwhile, Rajeswari et al., [3] analyzed the influence of chemical reaction parameter, magnetic parameter, buoyancy parameter and suction parameter on nonlinear MHD boundary layer flow through a vertical porous surface.

Radiative heat and mass transfer play an important role in manufacturing industries for the design of fins, steel rolling, nuclear power plants, and gas turbines. Various propulsion devices for aircraft, missiles, satellites and space vehicles are examples of such engineering applications. If the

\footnotetext{
*Corresponding author.

E-mail address: lavanya.b@manipal.edu (Bommanna Lavanya)
}

https://doi.org/10.37934/arfmts.68.1.110 
temperature of the surrounding fluid is rather high, radiation effects play an important role and this situation exists in space technology. In such cases, one has to take into account the effect of thermal radiation and mass diffusion. England and Emery [4] studied thermal radiation effects of an optically thin grey gas bounded by a stationary vertical plate. Radiation effects on mixed convection along an isothermal vertical plate were also studied by Hossain and Takhar [5]. Raptis and Perdikis [6] studied the effects of thermal radiation and free convection flow past a moving vertical plate, the governing equations were solved analytically. Das et al., [7] analyzed radiation effects on flow past an impulsively started infinite isothermal vertical plate.

The effects of chemical reaction and radiation absorption on free convective flow through a porous medium with a variable suction in the presence of uniform magnetic field were studied by Sudheer Babu and Satyanarayana [8]. Ahmed and Alam Sarker [9] presented the problem of a steady two - dimensional natural convective flow of a viscous incompressible and electrically conducting fluid past a vertical impermeable flat plate in the presence of a uniform transverse magnetic field. Saravana et al., [10] studied the effects of mass transfer on the MHD viscous flow past an impulsively started infinite vertical plate with constant mass flux. Lavanya [11] studied MHD rotating flow through a porous medium with heat and mass transfer. Few other related studies can be found in [12-16].

In the present paper, we extend our previous work by incorporating concentration equations with thermal radiation term to study hall current and thermal radiation effect on heat and mass transfer of unsteady MHD flow of a viscoelastic micropolar fluid through a porous medium. The governing Equations are solved analytically using perturbation method and effect of various physical parameters are discussed numerically and graphically.

\section{Methodology}

We consider the unsteady flow of a viscous incompressible and electrically conducting viscoelastic micropolar fluid over an infinite vertical porous plate, subjected to a constant transverse magnetic field $B_{0}$ in the presence of thermal and concentration buoyancy effects. The induced magnetic field is assumed to be negligible compared to the applied magnetic field. The $x$-axis is taken along the planar surface in the upward direction and the y-axis taken to be normal to it as shown in figure 1. Due to the infinite plane surface assumption, the flow variables are function of $y$ and $t$ only. The plate is subjected to a constant suction velocity $v_{0}$.

The governing equations of flow under the usual Boussinesq approximation are given by

$$
\begin{aligned}
& \frac{\partial v}{\partial y}=0 \\
& \frac{\partial u}{\partial t}+v \frac{\partial u}{\partial y}=\left(v+v_{r}\right) \frac{\partial^{2} u}{\partial y^{2}}-K_{0} \frac{\partial^{3} w}{\partial t \partial y^{2}}+v_{r} \frac{\partial N_{1}}{\partial y}+g \beta_{T}\left(T-T_{\infty}\right)+g \beta_{C}\left(C-C_{\infty}\right)-\sigma \frac{B_{0}^{2}(u+m w)}{\rho\left(1+m^{2}\right)}-\frac{v}{k} u \\
& \frac{\partial w}{\partial t}+v \frac{\partial w}{\partial y}=\left(v+v_{r}\right) \frac{\partial^{2} w}{\partial y^{2}}-K_{0} \frac{\partial^{3} w}{\partial t \partial y^{2}}+v_{r} \frac{\partial N_{2}}{\partial y}-\sigma \frac{B_{0}^{2}(w-m u)}{\rho\left(1+m^{2}\right)}-\frac{v}{k} w \\
& \rho J\left(\frac{\partial N_{1}}{\partial t}+v \frac{\partial N_{1}}{\partial y}\right)=\gamma \frac{\partial^{2} N_{1}}{\partial y^{2}}
\end{aligned}
$$




$$
\begin{aligned}
& \rho J\left(\frac{\partial N_{2}}{\partial t}+v \frac{\partial N_{2}}{\partial y}\right)=\gamma \frac{\partial^{2} N 2}{\partial y^{2}} \\
& \frac{\partial T}{\partial t}+v \frac{\partial T}{\partial y}=\frac{k}{\rho c_{p}} \frac{\partial^{2} T}{\partial y^{2}}-\frac{1}{\rho c_{p}} \frac{\partial q_{r}}{\partial y} \\
& \frac{\partial C}{\partial t}+v \frac{\partial C}{\partial y}=D \frac{\partial^{2} C}{\partial y^{2}}-k_{r}\left(C-C_{\infty}\right)
\end{aligned}
$$

The appropriate boundary conditions for the problem are

$$
\begin{aligned}
& u=L\left(\frac{\partial u}{\partial y}\right), \quad w=L\left(\frac{\partial u}{\partial y}\right), N_{1}=-n\left(\frac{\partial u}{\partial y}\right), N_{2}=-n\left(\frac{\partial w}{\partial y}\right), \\
& T=T_{\infty}+\left(T_{w}-T_{\infty}\right) e^{i w t}, \\
& C=C_{\infty}+\left(C_{w}-C_{\infty}\right) e^{i w t} \text { at } y=0 \\
& u \rightarrow 0, w \rightarrow 0 N_{1} \rightarrow 0, \\
& N_{2} \rightarrow 0, T \rightarrow T_{\infty}, C \rightarrow C_{\infty} \text { at } \quad y \rightarrow \infty
\end{aligned}
$$

where $u, v$ and $w$ are velocity components along $\mathrm{x}, \mathrm{y}$ and $\mathrm{z}$-axis respectively. $\mathrm{v}$ is the kinematic viscosity, $\mathrm{g}$ is the acceleration due to gravity, $\beta_{T}$ and $\beta_{c}$ are the coefficients of thermal expansion and concentration expansion respectively, $\mathrm{T}$ is the dimensional temperature of the fluid, $T_{w}$ and $T_{\infty}$ denotes the temperature at the plate and temperature far away from the plate respectively, $\mathrm{C}$ is the dimensional concentration of the solute, $C_{w}$ and $C_{\infty}$ are concentration of the solute at the plate and concentration of the solute far from the plate respectively, $\mathrm{K}$ is the permeability of the porous medium, $\mathrm{k}$ is the thermal conductivity of the medium, $\rho$ is the density of the fluid, $\mathrm{j}$ is the micro inertia density or micro inertia per unit mass, $\gamma$ is the spin gradient viscosity, $\mathrm{L}$ is the characteristic length, $\mathrm{w}$ is the dimensional frequency of oscillation, $\sigma$ is the electrical conductivity, $\mathrm{m}$ is the hall current parameter and $\mathrm{D}$ is the molecular diffusivity, $q_{r}$ is the radiative heat flux.

The constant that related to microgyration vector and shear stress is $\mathrm{n}$, where $0 \leq n \leq 1$. The case $n=0$ represents concentrated particle flows in which the microelement close to the wall surface are unable to rotate. thios case is also known as the strong concentration of microelements. the case $n=1$ is used for the vanishing of anti-symmetric part of the stress tensor and denotes weak concentration of microelements. the case $n=1$ is used for the modeling of turbulent boundary layer flows, we shall consider $\mathrm{n}=0$ and $\mathrm{n}=0.5$.

Following Rosseland approximation the radiative heat flux $q_{r}$ is modeled as

$$
q_{r}=\frac{4 \sigma}{3 k} \frac{\partial T^{4}}{\partial y}
$$

where $\sigma$ is the stefan-Boltzman constant and $\mathrm{k}$ is the mean absorption coefficient. Assuming that the difference in temperature within the flow are such that $T^{4}$ can be expressed as a linear combination of the temperature, we expand $T^{4}$ in taylor's series about $T_{\infty}$ as follows:

$T^{4}=T_{\infty}^{4}+4 T_{\infty}^{3}\left(T-T_{\infty}\right)+6 T_{\infty}^{2}\left(T-T_{\infty}\right)^{2}+--$

and neglecting higher order terms beyond the first degree in $\left(T-T_{\infty}\right)$, we have

$T^{4} \approx-3 T_{\infty}^{4}+4 T_{\infty}^{3} T$

Differentiating equation (9) with respect to y and using equation (11) to obtain 


$$
\frac{\partial q_{r}}{\partial y}=\frac{-16 T_{\infty}^{3} \sigma}{3 k} \frac{\partial_{2} T}{\partial y^{2}}
$$

Let us introduce the following dimensionless variables:

$$
\begin{aligned}
& u=\frac{u^{\prime}}{v_{0}}, \quad v=\frac{u^{\prime}}{v_{0}}, \quad w=\frac{w^{\prime}}{v_{0}}, \eta=\frac{v_{0} y^{\prime}}{v}, N_{1}=\frac{v N_{1}{ }^{\prime}}{v_{0}^{2}}, \quad N_{2}=\frac{v N_{2}{ }^{\prime}}{v_{0}^{2}}, \quad t=\frac{t^{\prime} V_{0}^{2} N_{1}}{4 v}, \quad w=\frac{4 v w^{\prime}}{v_{0}^{2}}, h=\frac{V_{0} L}{v} \\
& , \theta=\frac{T^{\prime}-T_{\infty}^{\prime}}{T^{\prime}{ }_{w}-T_{\infty}{ }^{\prime}}, \quad C=\frac{C^{\prime}-C^{\prime}{ }_{\infty}}{C^{\prime}{ }_{w}-C_{\infty}{ }^{\prime}} J=\frac{v_{0}{ }^{2} J^{\prime}}{v^{2}}
\end{aligned}
$$

Substituting Equation (13) into equations (2)-(8) yield the following dimensionless equations:

$$
\begin{aligned}
& \frac{1}{4} \frac{\partial u}{\partial t}-\frac{\partial u}{\partial \eta}=(1+\beta) \frac{\partial^{2} u}{\partial \eta^{2}}-a \frac{\partial^{3} u}{\partial t \partial \eta^{2}}+\beta \frac{\partial N_{1}}{\partial \eta}-\frac{M}{1+m^{2}}(m w+u)+G r \theta+G c C-\frac{u}{k} \\
& \frac{1}{4} \frac{\partial w}{\partial t}-\frac{\partial w}{\partial \eta}=(1+\beta) \frac{\partial^{2} u}{\partial \eta^{2}} w-a \frac{\partial^{3} w}{\partial t \partial \eta^{2}}-\beta \frac{\partial N_{2}}{\partial \eta}-\frac{M}{1+m^{2}}(w-m w)-\frac{u}{k} \\
& \frac{1}{4} \frac{\partial N_{1}}{\partial t}-\frac{\partial N_{1}}{\partial \eta}=L \frac{\partial^{2} N_{1}}{\partial \eta^{2}} \\
& \frac{1}{4} \frac{\partial N_{2}}{\partial t}-\frac{\partial N_{2}}{\partial \eta}=L \frac{\partial^{2} N_{2}}{\partial \eta^{2}} \\
& \frac{1}{4} \frac{\partial \theta}{\partial t}-\frac{\partial \theta}{\partial \eta}=\frac{1}{p r}\left(1+N_{r}\right) \frac{\partial^{2} \theta}{\partial \eta^{2}} \\
& \frac{1}{4} \frac{\partial C}{\partial t}-\frac{\partial C}{\partial \eta}=\frac{1}{S c} \frac{\partial^{2} C}{\partial \eta^{2}}-K_{r} C
\end{aligned}
$$

Where $\beta=v_{r} / v$ is the dimensionless viscosity ratio, $a=K_{0} v_{0}^{2} / 4 v^{2}$ is the viscoelastic parameter, $M=\sigma B_{0}^{2} v / \rho V_{0}^{2}$ is the magnetic field parameter, $N_{r}=16 T_{\infty}{ }^{\prime} \sigma^{\prime} / 3 k^{\prime} k_{\text {is }}$ the thermal radiation parameter, $G r=v \beta_{t} g\left(T_{w}{ }^{\prime}-T_{\infty}{ }^{\prime}\right) / V_{0}^{3}$ is the Grashof number, $G c=v \beta_{t} g\left(C_{w}{ }^{\prime}-C_{\infty}{ }^{\prime}\right) / V_{0}^{3}$ is the modified Grashof number, $p r=v \rho C p / k$ is the prandtl number is the schmidt number, $S c=v / D$ is the permeability of the porous medium parameter and $L=\gamma V_{0}^{2} / \rho v^{3} j$ is the material parameter Also the boundary conditions becomes

$$
\begin{aligned}
& u=h \frac{\partial u}{\partial \eta}, w=h \frac{\partial w}{\partial \eta}, \theta=e^{i w t}, C=e^{i w t}, N_{1}=-n \frac{\partial u}{\partial \eta}, N_{2}=-n \frac{\partial w}{\partial \eta} \quad \text { at } \quad \mathrm{y}=0 \\
& u \rightarrow 0, w \rightarrow 0, \theta \rightarrow 0, C \rightarrow 0, N_{1} \rightarrow 0, N_{2} \rightarrow 0, \text { at } y \rightarrow \infty \\
& \frac{1}{4} \frac{\partial q}{\partial t}-\frac{\partial q}{\partial \eta}=(1+\beta) \frac{\partial^{2} q}{\partial \eta^{2}}-a \frac{\partial^{3} q}{\partial t \partial \eta^{2}}+ \\
& i \beta \frac{\partial p}{\partial \eta}-\frac{M}{1+m^{2}}(1-i m) q+G r \theta+G c C-\frac{q}{k} \\
& \frac{1}{4} \frac{\partial p}{\partial t}-\frac{\partial p}{\partial \eta}=L \frac{\partial^{2} p}{\partial \eta^{2}}
\end{aligned}
$$


$\frac{1}{4} \frac{\partial \theta}{\partial t}-\frac{\partial \theta}{\partial \eta}=\frac{1}{p r}\left(1+N_{r}\right) \frac{\partial^{2} \theta}{\partial \eta^{2}}$

$\frac{1}{4} \frac{\partial C}{\partial t}-\frac{\partial C}{\partial \eta}=\frac{1}{S c} \frac{\partial^{2} C}{\partial \eta^{2}}-K_{r} C$

and the corresponding boundary conditions are

$q=h \frac{\partial q}{\partial \eta}, \theta=e^{i w t}, C=e^{i w t}, P=i n \frac{\partial q}{\partial \eta} \quad$ at $\quad y=0$

$q \rightarrow 0, \theta \rightarrow 0, C \rightarrow 0, p \rightarrow 0$, at $y \rightarrow \infty$

\subsection{Method of Solution}

In order to solve equations (21)-(24) subject to the boundary conditions (25), we assume a perturbation method of this form:

$q=q_{0}(\eta) e^{i w t}, \quad p=p_{0}(\eta) e^{i w t}, \quad \theta=\theta_{0}(\eta) e^{i w t}, \quad C=C_{0}(\eta) e^{i w t}$

\subsection{Calculation}

Substituting equation (26) in to equations (21)-(24), we obtain the following set of equations:

$\left(a_{1}-i a_{2}\right) q_{0}{ }^{\prime \prime}+q_{0}{ }^{\prime}-\left(a_{3}+i a_{4}\right) q_{0}=$

$G r \theta_{0}-G c C_{0}-i \beta p_{0}^{\prime}$

$L P_{0}^{\prime \prime}+P_{0}^{\prime}-\frac{i w}{4} p_{0}=0$

$a_{5} \theta_{0}^{\prime \prime}+\theta_{0}^{\prime}-\frac{i w}{4} \theta_{0}=0$

$C_{0}^{\prime \prime}+S c C_{0}^{\prime}-\frac{K r S c i w}{4} C_{0}=0$

where $a_{1}=1+\beta, a_{2}=a \omega, \quad a_{3}=M / 1+m^{2}+1 / k, a_{4}=(\omega / 4)-\left(M m^{2} / 1+m^{2}\right), \quad a_{5}=(1+N r) / \operatorname{Pr}$ The corresponding boundary conditions can be written as

$q_{0}=h \frac{\partial q_{0}}{\partial n}, \quad \theta_{0}=1, \quad C_{0}=1, \quad p_{0}=i n \frac{\partial q_{0}}{\partial n}, \quad$ at $\mathrm{y}=0$

$q_{0}=0, \quad \theta_{0}=0, \quad C_{0}=0, \quad p_{0}=1, \quad$ at $\quad y \rightarrow \infty$,

The solution of Eqs. (27)-(30) satisfying the boundary conditions 31 are given by thickness which is expected since when the holes porous medium

$q=\left(A_{4} e^{-m 4 y}+A_{1} e^{-m 2 y}+A_{2} e^{-m 1 y}+A_{3} e^{-m 3 y}\right) e^{i \omega t} p=B_{1} e^{i w t-m 3 y}$

$\theta=e^{i w t-m 2 y}$

$C=e^{i w t-m l y}$

The skin-friction, Nusselt number and Sherwood number are important physical parameters for this type of boundary layer flow. these parameters can be defined and determined as follows. 
$C_{f}=\left(\frac{\partial q}{\partial y}\right)_{y=0}=\left(m_{4} A_{4}+m_{2} A_{1}+m_{1} A_{2}+m_{3} A_{3}\right) e^{i \omega t}$

The rate of heat transfer at the surface in terms of nusselt number is given by

$N u=-\left(\frac{\partial \theta}{\partial y}\right)_{y=0}=m_{2} e^{i \omega t}$

The rate of mass transfer at the surface in terms of the local sherwood number is given by

$S h=-\left(\frac{\partial C}{\partial y}\right)_{y=0}=m_{3} e^{i \omega t}$

\section{Results and Discussions}

The results are presented as velocity, temperature and concentration profiles in the below graphs. The effects of magnetic field parameter on velocity distribution profiles across the boundary layer are presented in Fig-1. It is obvious that the effect of increasing values of the magnetic field parameter $\mathrm{M}$ results in a decreasing velocity distribution across the boundary layer. This is due to fact that the effect of a transverse magnetic field give rise to a resistive type force called the lorentz force. The force has the tendency to slow the motion of the fluid.

Figure 2 depicts the effects of permeability of the porous medium parameter( $k)$ on velocity distribution profiles and it is obvious that as permeability parameter ( $k$ ) increases, the velocity increases along the boundary layer become larger, the resistivity of the medium may be neglected.

Figure 3 shows the translation velocity profiles with different values of radiation parameter and the effect of increasing the radiation parameter is to increase the translational velocity. This is because when the intensity of heat generated through radiation increased, the bond holding the components of the fluid particles is easily broken and the fluid velocity will increase.

Figure 4 displays the effect of Hall current parameter on the translational velocity distribution profiles. It is noticed that the hall current parameter increases the velocity. Figures 5 and 6 illustrates the effect of parameter on micro rotational velocity profiles. The profiles increase as the parameter increases and presents the effect of the Prandtl number $(P r)$ on the temperature profiles. Increasing the value of pr has the tendency to decrease the fluid temperature in the boundary layer as well as the thermal boundary layer thickness. This causes the wall slope of the temperature to decrease as pr is increasing as causing the Nusselt number to increase as can be clearly seen.

The numerical result of skin friction, Nusselt number and Sherwood number are shown in the tables 1-2. Table 1 shows the effect of $P r$ and radiation parameter $(\mathrm{N})$ on the Nusselt number increases as $P r$ increases. This shows that the surface heat transfer from the porous plate increases with the increasing values of $\operatorname{Pr}$ and decreases with increasing value of $\mathrm{N}$. Table 2 shows that the effect of increasing the $\mathrm{Sc}$ is to increases the nusselt number also increases. 


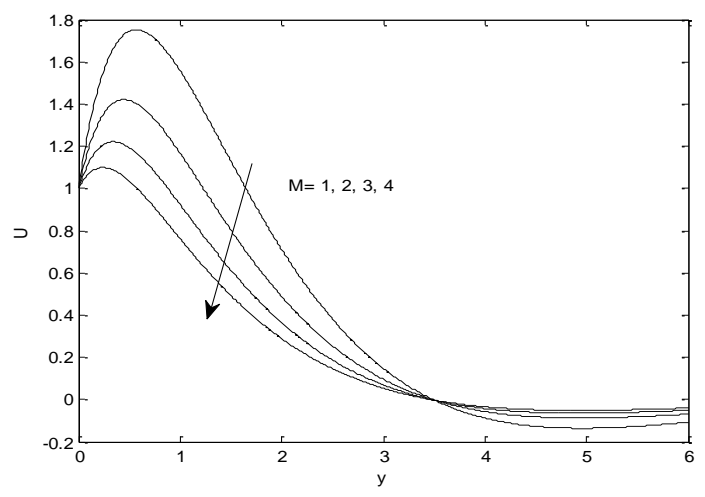

Fig. 1. Effects of magnetic parameter on velocity Profiles

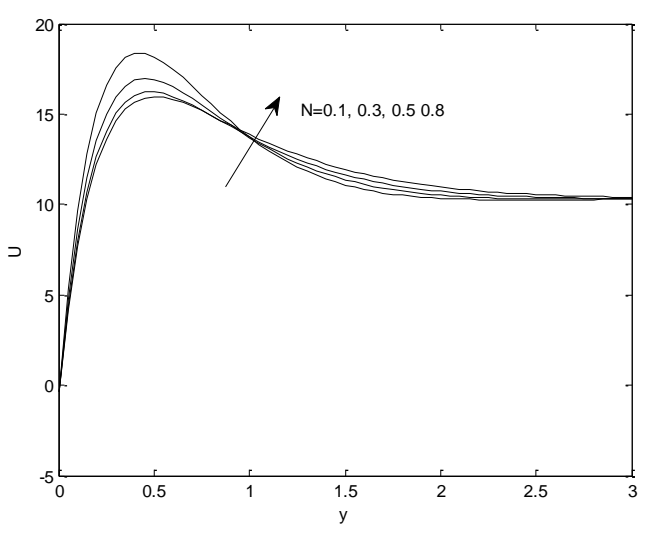

Fig. 3. Velocity profiles for different values of $\mathrm{N}$

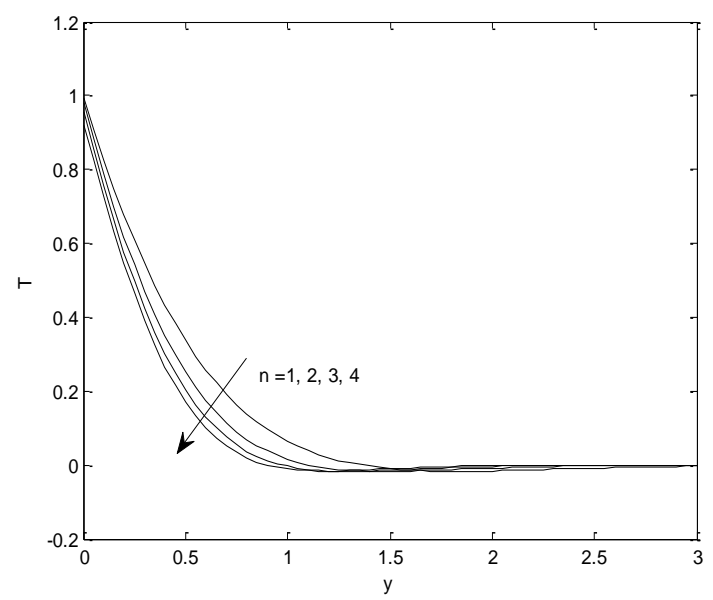

Fig. 5. Temperature profiles for different values of $n$

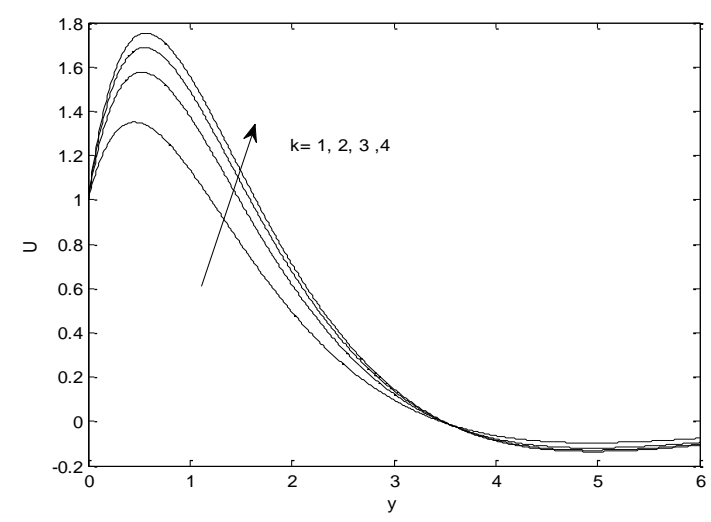

Fig. 2. Effects of permeability parameter on velocity profiles

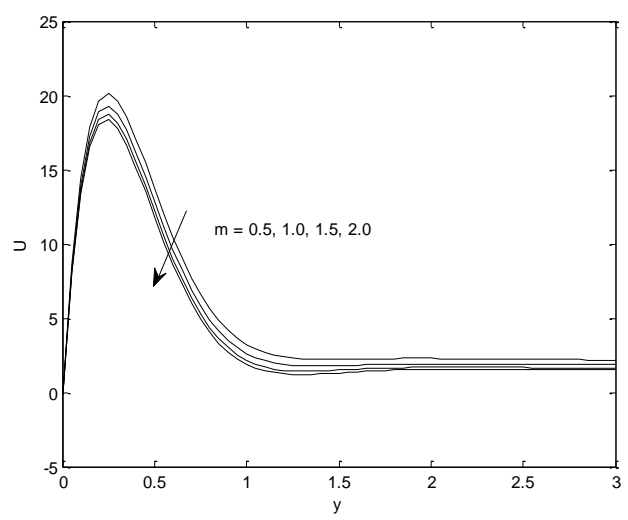

Fig. 4. Velocity profiles for different values of $m$

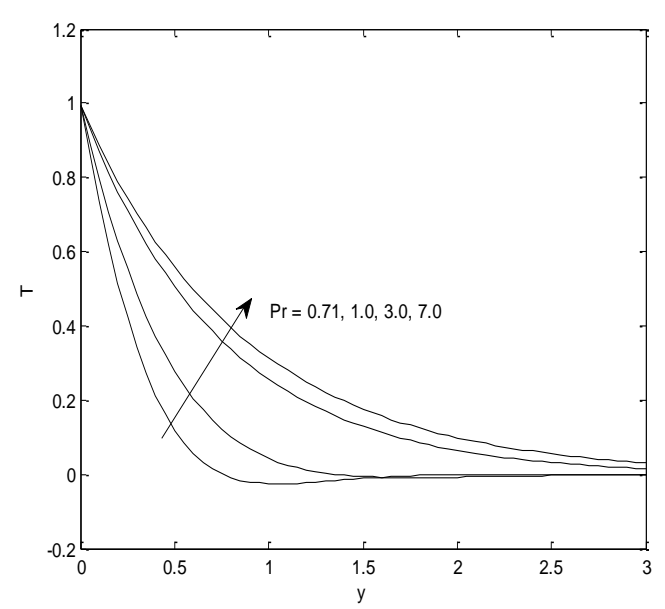

Fig. 6. Temperature profiles for different values of $\mathrm{Pr}$ 


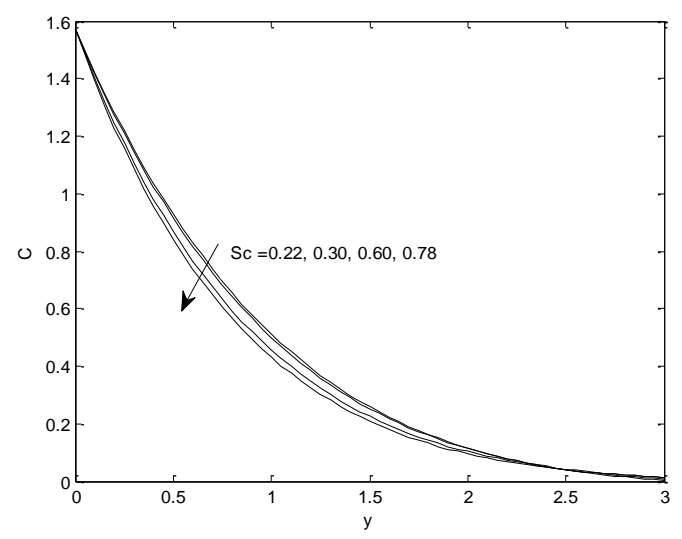

Fig. 7. Concentration profiles for different values Schmidt number

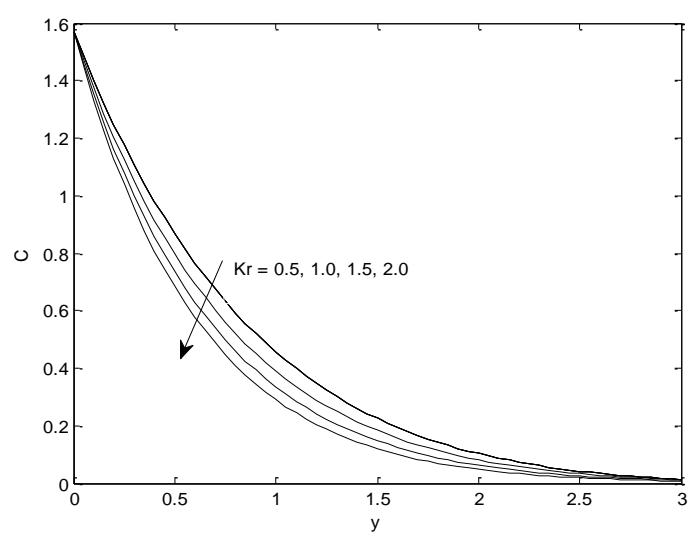

Fig. 8. Concentration profiles for different values of chemical reaction parameter

Table 1

Effects of $\mathrm{Pr}$ and $\mathrm{N}$ on Nusselt number $\mathrm{Nu}$

\begin{tabular}{ccc}
\hline$P r$ & $N$ & $N u$ \\
\hline 3 & 0.5 & 1.9661 \\
4 & 0.5 & 2.6176 \\
5 & 0.5 & 3.2716 \\
3 & 0.2 & 2.4544 \\
3 & 0.4 & 2.1054 \\
3 & 0.8 & 1.6421 \\
\hline
\end{tabular}

\section{Table 2}

Effects of Sc on Sherwood number, Sh

\begin{tabular}{ll}
\hline Sc & $N u$ \\
\hline 2 & 1.9661 \\
3 & 2.9444 \\
4 & 3.9271 \\
\hline
\end{tabular}

\section{Conclusions}

The study of MHD heat and mass transfer flow of an incompressible, electrically conducting viscoelastic micropolar fluid over an infinite vertical plate through porous medium was conducted. The results are discussed through graphs and tables for different values of parameters. Following conclusions can be drawn from the results obtained:

- The Nusselt number increased as the prandtl number increased and decreased as radiation parameter increased.

- The Sherwood number increased as the Schmidt number increased.

- In the presence of a uniform magnetic field, increase in the strength of the applied magnetic field decelerated the fluid motion along the wall of the plate inside the boundary layer. 
- Increase in hall current parameter increases the momentum and thermal boundary layer thickness.

- The radiation parameter increases both skin friction coefficient and couple stress coefficient.

\section{Appendix:}

$$
\begin{gathered}
m_{1}=\frac{-S c+\sqrt{S c^{2}+i \omega S c}}{2}, \quad m_{2}=\frac{-1+\sqrt{1+a_{5} i \omega}}{2} \quad, \quad m_{3}=\frac{-1+\sqrt{1+4 \frac{L i \omega}{4}}}{2}, \\
T_{1}=a_{1}-i a_{2}, \quad T_{2}=a_{3}-i a_{4}, \quad m_{4}=\frac{-1+\sqrt{1+4 T_{1} T_{2}}}{2 T_{1}}, \quad A_{1}=\frac{-G r}{T_{1} m_{2}^{2}-m_{2}-\left(a_{3}+i a_{4}\right)} \\
A_{2}=\frac{-G c}{T_{1} m_{1}^{2}-m_{1}-\left(a_{3}+i a_{4}\right)}, \quad A_{3}=\frac{-\beta n m_{3} T}{T_{1} m_{3}^{2}-m_{3}-\left(a_{3}+i a_{4}\right)}, \quad A_{4}=h T-A_{1}-A_{2}-A_{3}
\end{gathered}
$$

\section{References}

[1] Khaleque, Tania S., and M. A. Samad. "Effects of radiation, heat generation and viscous dissipation on MHD free convection flow along a stretching sheet." Research Journal of Applied Sciences, Engineering and Technology 2, no. 4 (2010): 368-377.

[2] Amkadni, Maryem, and Adnane Azzouzi. "On a similarity solution of MHD boundary layer flow over a moving vertical cylinder." International Journal of Differential Equations $2006 \quad$ (2006). https://doi.org/10.1155/DENM/2006/52765

[3] Rajeswari, R., B. Jothiram, and V. K. Nelson. "Chemical reaction, heat and mass transfer on nonlinear MHD boundary layer flow through a vertical porous surface in the presence of suction." Applied Mathematical Sciences 3, no. 4952 (2009): 2469-2480.

[4] England, W. G., and A. F. Emery. "Thermal radiation effects on the laminar free convection boundary layer of an absorbing gas." (1969): 37-44. https://doi.org/10.1115/1.3580116

[5] Hossain, M. A., and H. S. Takhar. "Radiation effect on mixed convection along a vertical plate with uniform surface temperature." Heat and Mass transfer 31, no. 4 (1996): 243-248. https://doi.org/10.1007/BF02328616

[6] Raptis, A., and C. Perdikis. "Radiation and free convection flow past a moving plate." Applied Mechanics and Engineering 4, no. 4 (1999): 817-821.

[7] Das, U. N., R. K. Deka, and V. M. Soundalgekar. "Radiation effects on flow past an impulsively started vertical infinite plate." J. theo. Mech 1 (1996): 111-115.

[8] Sudheer Babu, M., and P. V. Satya Narayana. "Effects of the chemical reaction and radiation absorption on free convection flow through porous medium with variable suction in the presence of uniform magnetic field." Journal of Heat and mass transfer 3 (2009): 219-234.

[9] Ahmmed, S. F., and M. S. Sarker. "MHD natural convection flow of viscous incompressible fluid from a vertical flat plate." (2009).

[10] Saravana, R., S. Sreekanth, S. Sreenadh, and R. Hemadri Reddy. "Mass transfer effects on MHD viscous flow past an impulsively started infinite vertical plate with constant mass flux." Advances in Applied Science Research 2, no. 1 (2011): 221-229.

[11] Lavanya, Bommanna. "MHD Rotating Flow Through a Porous Medium with Heat and Mass Transfer." Journal of Advanced Research in Fluid Mechanics and Thermal Sciences 54, no. 2 (2019): 221-231.

[12] Nagasasikala, Madduleti, and Bommanna Lavanya. "Effects of Dissipation and Radiation on Heat Transfer Flow of a Convective Rotating Cuo-Water Nano-fluid in a Vertical Channel." Journal of Advanced Research in Fluid Mechanics and Thermal Sciences 50, no. 2 (2018): 108-117.

[13] Nagasasikala, Madduleti, and G. Phrabhakar Rao. "Heat and mass transfer of a MHD flow of a nanofluid through a porous medium in an annular, circular region with outer cylinder maintained at constant heat flux." CFD Letters 11, no. 9 (2019): 32-58.

[14] Umair, Siddique Mohd, Emaad Ansari, Sher Afghan Khan, and Abdulrahman Alrobaian. "On Numerical Investigation of Semi-Empirical Relation Representing Development Length for a Fluid Flow in a Closed Conduit." CFD Letters 11, no. 6 (2019): 63-71. 
[15] Muhammad Arif Harun, Prem Gunnasegaran, Nor Azwadi Che Sidik. "Experimental Investigations of Loop Heat Pipe Performance with Nanofluids." Journal of Advanced Research Design 52, no. 1 (2019): 13-27

[16] Khattak, Muhd Adil, Abdul Hakim Ali, Muhammad Firdaus Talhah, Fakruddin Mustakim Alisah, Chin Kim Wei, Rustam Khan, and Sajjad Tahir. "Radiation protection, design safety and material aspects of nuclear power plants: A review." Journal of Advanced Research in Materials Science 38, no. 1 (2017): 1-14. 